\title{
Performance Analysis of Genetic Zone Routing Protocol Combined With Vertical Handover Algorithm for 3G-WiFi Offload
}

\author{
Setiyo Budiyanto, Muhammad Asvial \& Dadang Gunawan \\ Department of Electrical Engineering \\ Faculty of Engineering, University of Indonesia, Depok 16424, Indonesia \\ E-mail: setiyo.budiyanto21@ui.ac.id
}

\begin{abstract}
In the deployment scenario of multiple base stations there is usually a deficiency in the routing protocols for load balancing in the wireless network. In this study, we propose a routing algorithm that can be implemented inMobile Adhoc Networks (MANETs) as well as third-generation (3G)-Wireless Fidelity (WiFi) offload networks. We combined the Genetic Zone Routing Protocol (GZRP) with the Vertical Handover (VHO) algorithm in a 3G-WiFi offload network with multiple base stations. Simulation results show that the proposed algorithm yields improvement in the received signal strength (which is increased up to $25 \mathrm{dBm}$ ), user throughput (which is approximately $1 \mathrm{Mbps}-2.5 \mathrm{Mbps}$ ), and data rate (which is increased up to $2.5 \mathrm{Mbps}$ ).
\end{abstract}

Keywords: 3G-WiFi offload; GZRP; load balancing; mobile ad-hoc network; VHO.

\section{$1 \quad$ Introduction}

Data traffic has increased by $131 \%$ since 2011 [1]. This has encouraged some mobile operators in Europe to invest in machine-to-machine communications. However, the existing mobile networks are not able to accommodate the exponential growth in data traffic. As a result, traffic offload may arise. One way to overcome offload is to employ a network architecture called Metropolitan Advanced Delivery Networks (MADNETs).

MADNETs provided an integrated solution for the management of cellular traffic in [2]. Specifically, femtocells were deployed in indoor environments; WiFi was used in outdoor environments; and peer-to-peer offload was considered in mobile environments. MADNETs allow mobile WiFi networks to have remarkable coverage and downloading and uploading speeds [2],[3]. In addition, an algorithm is available that is able to provide an improved handover process of UMTS/3G to WiFi, known as Vertical Handover (VHO) [4],[5]. Moreover, in $3 \mathrm{G}$ and $\mathrm{WiFi}$ offload networks there are no specific routing protocols for both dynamic and static load balancing to control traffic when one node is either turned off or in full traffic.

Received October $19^{\text {th }}, 2013,1^{\text {st }}$ Revision March $22^{\text {nd }}, 2014,2^{\text {nd }}$ Revision April $7^{\text {th }}, 2014,3^{\text {rd }}$ Revision April $28^{\text {th }}, 2014$, Accepted for publication April $29^{\text {th }}, 2014$.

Copyright (c) 2014 Published by ITB Journal Publisher, ISSN: 2337-5787, DOI: 10.5614/itbj.ict.res.appl.2014.8.1.4 
On the other hand, WiFi networks have a separate system architecture that applies to high-speed WiFi networks, called Mobile Adhoc Networks (MANETs). MANETs have some autonomous nodes that are able to arrange themselves in various ways and operate without strict top-down network administration. A hybrid protocol for MANETs, called the Zone Routing Protocol (ZRP), was proposed in [6] in order to reduce the control overhead of the proactive routing protocols and decrease the latency caused by route discovery in the reactive routing protocols. However, the actual problem emerges when the destination is outside the zone. In this case, we can make use of route discovery with the Interzone Routing Protocol (IERP) [7] or the Bordercast Resolution Protocol (BRP) [8]. This route discovery applies to the border nodes between the zones. At this point, a genetic algorithm (GA) can be implemented [9]: each border node implements the GA to find multiple shortest or nearly shortest paths. Instead of rediscovering the path to the destination every failure time of the existing path, the border node will make use of the available shortest or nearly shortest paths. In this paper, we will discuss implementation of the Genetic Zone Routing Protocol (GZRP) in 3G-WiFi offload technology.

GZRP on MANETs is used as a load-balancing algorithm that provides the total packets received with some alternative routes in order to reduce traffic load on a single route. The proposed algorithm combines the GZRP with the VHO algorithm as a routing protocol on $3 \mathrm{G}-\mathrm{WiFi}$ offload networks with multiple base stations. It distributes traffic to multiple neighbor nodes in the network to provide some alternative paths when traffic flows into each node. As a result, the 3G-WiFi offload network increases the throughput of the mobile subscriber (MS) and reduces the duration of handover. Moreover, the proposed algorithm greatly improvesthe received signal strength (RSS) at the MS. Good signal quality at the MS provides improvement of the data rate. In addition, a declining data rate affected by an increase in the number of users can be overcome. Thus, the proposed algorithm reduces the amount of traffic going into a bottleneck and minimizes handover failures and call drop occurrence.

We can summarize the contributions of this paper as follows:

1. We combine the GZRP and the VHO algorithm as a routing protocol on 3G-WiFi Offload.

2. We will show that some improvements in the received signal strength, user throughput, and data rate are achievable by using the proposed method.

3. We will also show that our algorithm is robust under an increase of the number of users, as it maintains the data rate and minimizes handover failures. 
The rest of this paper is organized as follows. In Section 2, we briefly discuss related works. In Section 3, we present the proposed algorithm and also its mathematical model. In Section 4, we present and analyze the results of a simulation. Finally, the conclusions are provided in Section 5.

\section{$2 \quad$ Related Works}

The GZRP, an extension of the Zone Routing Protocol (ZRP) by adopting a genetic algorithm, was proposed in [10]. Previous work related to GZRP performance improvement has been done: for scalability of the size of MANETs [11]; application of genetic algorithms contained in the GZRP in designing security models on MANETs [12]; and evaluation of GZRP performance on MANETs [13].

In [14], a function-arranging vertical handover uses a two-step handover decision in heterogeneous wireless mobile networks, known as the Vertical Handover Decision Function (VHDF) algorithm. In [15], an improved VHDF algorithm is discussed, which is called the Extended Vertical Handover Decision Function (EVHDF) algorithm and uses the Dynamic New Call Blocking Probability (DNCBP) algorithm. DNCBP is more dynamic than NCBP regarding the ability to find out the traffic load in a network. In [4], the vertical handover was focused on goodput and RSS, and the authors experimented with a hybrid RSSI algorithm.

However, the algorithms in [4] and [15] still have a drawback when the network encounters a capacity and/or user increase: the more users access a node, the lower the node quality. Thus, there is a need of a load balancing traffic solution in order to share the traffic in a node acquiring full traffic. The algorithm proposed in [15] was only able to find out traffic conditions in each node, but it could not maximize the load balancing in all the nodes of the network. In [16] and [17], the GZRP was able to improve the efficiency of the network, as well as reduce the burden on the path in order in order to balance the distribution of packet delivery through available alternative paths. By using the GZRP in MADNETs, there is an expectation to find the best solution for load balancing problems in $3 \mathrm{G}-\mathrm{WiFi}$ offload networks with multiple base stations.

\section{Proposed Algorithm and its Mathematical Model}

The network architecture adopting the GZRP concept is a combination of the $3 \mathrm{G}$ network and the WiFi offload network architectures. More specifically, connection to the Internet is realized via either the Gateway GPRS Support Node (GGSN, an Internet gateway for 3G (UMTS mobile traffic)), when the user is in a $3 \mathrm{G}$ network, or the Service Control Engine (SCE), when the user is 
in a WiFi network. There is an emphasis in GZRP routing on GGSN functions as regulators of the traffic load balancing, which is carried out to identify its access towards the side of the Radio Network Controller (RNC), for UMTS 3G networks (3G), or the Wireless Access Gateway (WAG), for WiFi networks. In GGSN, there is an algorithm that controls the process of handover from $3 \mathrm{G}$ to WiFi.

In our proposed algorithm, the GZRP concept is implemented in the WiFi offload gateway and the GGSN. The 3G-WiFi offload network enables the data to equalize the traffic side of the base station with direct instructions from the gateway in the form of an algorithm that updates the results of a combination of existing algorithms using the GZRP.

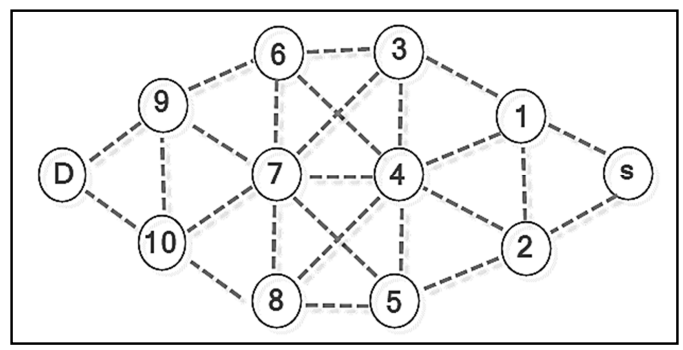

Figure 1 Dynamic Routing Graph.

In this research, the Dynamic Routing concept, as shown in Figure 1, was implemented. In the case of the GZRP, when the source node (depicted as S) reinitiates the neighbor nodes in the network, it will find a bandwidth limitation on the destination node (depicted as D) and a power condition that is low or at the minimum threshold. We expect that the GZRP can maintain the network's stability when traffic handover is being performed. For example, when a neighbor node in the WiFi network cannot handle the bandwidth of the MS, the traffic in the neighbor node will first balance its load with all its neighbors at the same gateway, using the GZRP. This also happens when the power condition of the neighbor node becomes low, so the Wireless Access Gateway will use the GZRP to give the information signal to the nearest neighbor node with the best coverage for the mobile subscriber. Thus, it can minimalize handover time.

\subsection{First Step: Initiation}

A pre-handover decision is employed to evaluate if the requirements for executing handover are supported in every $\mathrm{x}$ network, where $\mathrm{x}=1,2, \ldots, \mathrm{N}$, and $\mathrm{N}$ is the number of different networks in one area of coverage. The mathematical equation for the handover process uses step functions as shown in 
Eq. (1). The equation shows that $\mathrm{Mx}$ is a function of the following required parameters: bandwidth (b), received signal strength (RSS), velocity (V), battery power $(\mathrm{P})$, duration $(\mathrm{T})$, and traffic cost $(\mathrm{C})$. Since the step function, $\mathrm{f}(\mathrm{x})$, has value 0 for $x<0$ and 1 for $x>1$, it is clear that if one or more parameters are below the threshold, Mx will be 0 [15].

$$
\begin{aligned}
& M_{x}=f\left(b_{x}-b_{t h}\right) \cdot f\left(R S S_{x}-R S S_{t h}\right) \cdot f\left(V_{x}-V_{t h}\right) \cdot f\left(T_{x}-\right. \\
& \left.T_{t h}\right) \cdot f\left(P_{x}-P_{t h}\right) \cdot f\left(C_{x}-C_{t h}\right)
\end{aligned}
$$

In this research, we focused on the battery power and bandwidth parameters in the neighbor nodes (NNs). When $f\left(P_{x}-P_{t h}\right)$ or $f\left(b_{x}-b_{t h}\right)$ is 0 (zero), the GZRP carries out the process. In a condition where all the parameters in $M_{x}$ have value 1 (one), the network will be added to the network candidates given by S. If MN lacks resource nodes and the current network is in the set $S$, MN will remain connected to the current network. If $\mathrm{MN}$ is a resource-poor node and the current network is not in the set $S$, MN will handoff to any network included in the set $S$. If $\mathrm{MN}$ is a resource-rich node, $\mathrm{MN}$ will proceed to the second step.

\subsection{Second Step: Vertical Handoff Decision Process}

In this step, we use the Genetic Zone Vertical Handover Decision Function (GZVHDF), which is the Vertical Handoff Decision Function (VHDF) [14] using a distance vector as the network optimization tool [15], focusing on repairing the network condition, symbolized by $D_{j}$. In [15], Extended Vertical Handoff Decision Function (EVHDF), which is a combination of Dynamic New Call Blocking Probability and VHDF, was proposed. The EVHDF produces

$D_{j}=\frac{b_{j}}{H_{j}}$, where $b_{j}$ is the condition of the existing network bandwidth and $H_{j}$ is the value of DNCBP. In the EVHDF algorithm, repairing of the system takes place on the call blocking condition dynamically. But there is still a weakness when the network experiences bottleneck traffic on each node, which makes dynamic load balancing necessary. Therefore, when a neighbor node experiences full traffic load or even when power is under the minimum threshold, traffic will search for an available resource node to give the best service for the mobile subcriber to communicate in the network. The search for this resource node needs an algorithm that is capable of providing the best routing from source node to destination node. In this research, we used the GZRP algorithm, whose routing protocol hybrid is very suitable for load balancing at the gateway's side.

The mathematical equation for application of the GZRP is as follows: 
1. $\sum Y_{n}=$ Total cost; 'n' neighbor nodes that were passed from the source node (SN) to the destination node (DN), which is divided into several sections that allow traffic to reach the DN

2. $Z_{n}=$ Track that is taken from the lowest calculation of each $Y_{n}$

3. $f(X)=$ Value of each parameter that effects the handover destination node condition of the existing network, which is taken from the calculation of $Z_{n}$

Therefore, the network condition $\left(D_{j}\right)$ results in the following equation:

$$
D_{j}=\frac{b_{j}}{H_{j}}-\left[\left(\left(\frac{\sum Y_{n}-f(x)}{\sum Y_{n}}\right) \times 100 \%\right) \times \frac{b_{j}}{H_{j}}\right]
$$

So that the mathematical equations for the GZVHDF are:

$$
\begin{aligned}
& G Z Q_{j}=\frac{w_{c}\left(1 / C_{j}\right)}{\max \left(1 / C_{1}, \ldots, 1 / C_{m}\right)}+\frac{w_{s} S_{j}}{\max \left(S_{1}, \ldots, S_{m}\right)}+\frac{w_{p} P_{j}}{\max \left(P_{1}, \ldots, P_{m}\right)}+ \\
& \frac{w_{D} D_{j}}{\max \left(D_{1}, \ldots, D_{m}\right)}+\frac{w_{F} F_{j}}{\max \left(F_{1}, \ldots, F_{m}\right)}
\end{aligned}
$$

Thus, Eq. (6) has value $D_{j}$ from the calculation of Eq. (5), so the result of this $G Z Q_{j}$ givesthe best choice for the user to perform handover.

The proposed algorithm is detailed as follows:

Step 1: Identify the parameter of the VHO algorithm for heterogeneous mobile wireless networks. The required parameters are as follows :

$B \quad=$ Threshold current available bandwidth

RSS = Threshold received signal strength

$V \quad=$ Threshold velocity of mobile station

$T \quad=$ Threshold estimated time MS will be in present network

$P \quad=$ Threshold battery power of MS

$C \quad=$ Threshold cost of network for MS

Step 2: Create a rule for the value associated with the handoff parameters listed in Step 1 in order to determine the process (VHO) [15]

1) Handover will occur if the parameters - when connected to a WiFi or $3 \mathrm{G}$ network - are greater than $P_{x}^{T H}$.

2) Otherwise, theMS will stay connected to the same network and continue to Step 3

Step 3: Identify the neighboring base stations / access points

Step 4: Input the threshold values of the various parameters associated with the GZRP routingwhen prompted.

Step 5: Create rules for the parameter values associated with the GZRP in Step 3 to determine the GZRP routing process 
1) If the value is less than or equal to the $P_{x}^{T H}$ value then return to Step 4

2) If the value is greater than the $P_{x}^{T H}$ value then proceed to Step 6

Step 6: Apply the GZRP process [17],[18] as shown in Eq. (6)

If the RSS of WiFi exceedes low-threshold quality, the connection will be returned to the MS WiFi access point (AP). If the WiFi AP capacity is fully used, cellular traffic is connected in a cluster by using GZRP routing. When the current AP is already full, the MS will be transferred to the nearest AP. Thus, the implementation of the GZRP concept is intended to overcome the bottleneck and minimize connection drops due to the APs (base stations) being connected to mobile subscribers who experience full traffic.

\section{$4 \quad$ Simulation Results and Discussion}

\subsection{Simulation Settings}

We have applied the same parameters as in [4] for simulating the RSS, handover time and probability blocking, using simulator NS2 and Matlab. The simulation parameters are shown in Table 1.

Table 1 Simulation Parameters.

\begin{tabular}{lc}
\hline \multicolumn{2}{c}{ Data Simulations } \\
\hline Number of WiFi Access Points & 3 \\
Number of 3G Base Stations & 2 \\
Size of the Service Area & TCP \\
Traffic Type & \\
Transmission Range: & $600 \mathrm{~m}$ \\
3G Base Station & $100 \mathrm{~m}$ \\
WiFi Access Point & \\
Access Speed: & $15 \mathrm{Mbps}$ \\
3G Base Station & $8 \mathrm{Mbps}$ \\
WiFi Access Point & $5 \mathrm{~m} / \mathrm{s}$ \\
Maximum Speed &
\end{tabular}

We performed the simulation in two stages: first, VHO was used for arranging the process of handover for the $3 \mathrm{G}-\mathrm{WiFi}$ Offload; subsequently, the existing cellular signal conditions that will be simulated must be known. Secondly, the proposed algorithm was applied. We implemented the algorithm with a test download file of $10 \mathrm{MB}$ on a remote server (hosted by Google) using the HTTP over TCP protocol. The performance of GZRP routing on 3G-WiFi Offload improved the user's RSS, data rate, and throughput node as discussed in the following subsection. 
For the simulation we used MATLAB software with a set of functions called RUNE [19]. We used a wireless propagation model for the received signal (in $\mathrm{dB})$ as follows:

$$
P(d)=P_{t}+G+f_{i}(d)+S(d)
$$

and

$$
G=\alpha-10 \beta \log (d)
$$

where $P_{t}$ is effective transmitted power, $d$ is distance to transmitter, $\alpha$ is the path loss constant used to model the effect of antenna size and other physical parameters. The $\beta$ parameter determines how much the power decays as a function of distance from the base station. For free space propagation this parameter is 2 and in a typical urban environment it ranges from 3 to $4 . f_{i}(d)$ is a log-normal shadow fading factor used to model large-scale fluctuations of signal power caused by large obstacles (like hills or buildings) in the direction of radio wave propagation.

Moreover, we found out the handover time. The mathematical equations for the handover time in the proposed algorithm are as follows [20]:

\section{Step 1: Handover Time for Scan}

a. Horizontal scan

$$
t_{L 2 p-s c n(\max )}=N_{p-n b r} \times \gamma_{p}
$$

where $N_{p-n b r}$ comes from total transmission cost from MS to GGSN for the ongoing network and $\gamma_{p}=$ the number of neighbor AP/BTS to scan for the communication system (network source)

b. Vertical scan

$$
t_{L 2 n-s c n(a v g)}=N_{n-n b r} \times \gamma_{n}
$$

where $N_{n-n b r}$ comes from total transmission cost from MS to GGSN for the targeted network and $\gamma_{n}=$ the number of neighbor AP/BTS to scan for the communication system (network destination)

Step 2: Handover time for the reactive mode

$$
t_{\text {reactive }}=t_{F B U}+t_{F B a c k}
$$

where $t_{F B U}$ is time to send an FBU (Fast Binding Update) message and $t_{F B a c k}$ is time to accept feedback to/from the previous node. 
Step 3: Handover execution delay is given by

$$
t_{h n}^{*}=\theta_{n}=\left\{\begin{array}{c}
t_{A A A}=\theta_{W i F i} \\
t_{k e y}+t_{r a n g}+t_{r e g}+t_{c a p}=\theta_{3 G}
\end{array}\right.
$$

where $\theta_{n}$ is the total time of communication process initialization on each network, including:

1. AAA (WiFi)

2. Synchronization \&ranging $(3 \mathrm{G})$

3. Registration time $(3 \mathrm{G})$

4. Key exchange \& authorization $(3 \mathrm{G})$

5. Basic capability negotiation (3G)

Step 4. Total handover time + load balancing GZRP

$$
t_{H O}=t_{L 2 p-s c n(\max )}+t_{L 2 n-s c n(a v g)}+t_{\text {reactive }}
$$

In this research, we combined $g(x)$ with the GZRP because time $g(x)$ is related to the connection between the source node and the destination node in its calculation. Therefore, the following handover time formula is obtained:

$$
g(x)=t_{H O}-\left[\left(\left(\frac{\sum \gamma_{x}-f(x)}{\sum \gamma_{x}}\right) \times 100 \%\right) \times t_{H O}\right]+t_{h n}^{*}
$$

\subsection{Results and Discussions}

The simulation results for a system with VHO only and the proposed algorithm are shown in Figure 2 and Figure 3, respectively.

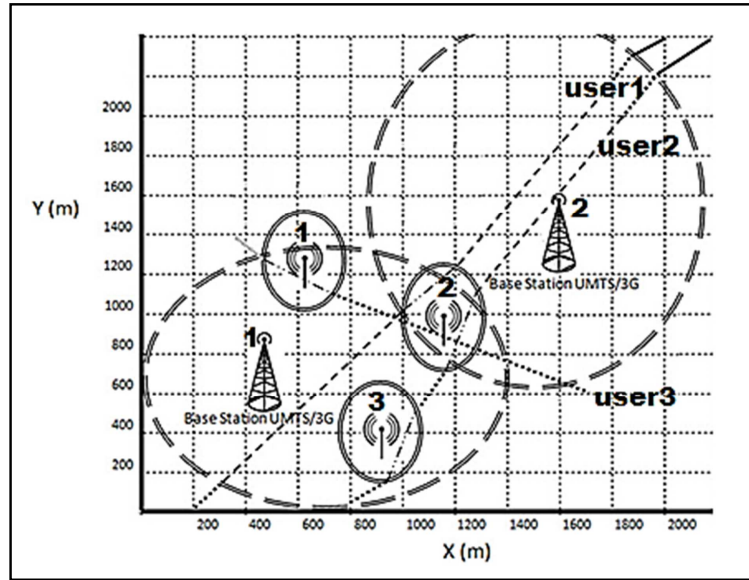

Figure 2 Simulation results $3 \mathrm{G}-\mathrm{WiFi}$ Offload with multiple base stations (VHO only) (*). 


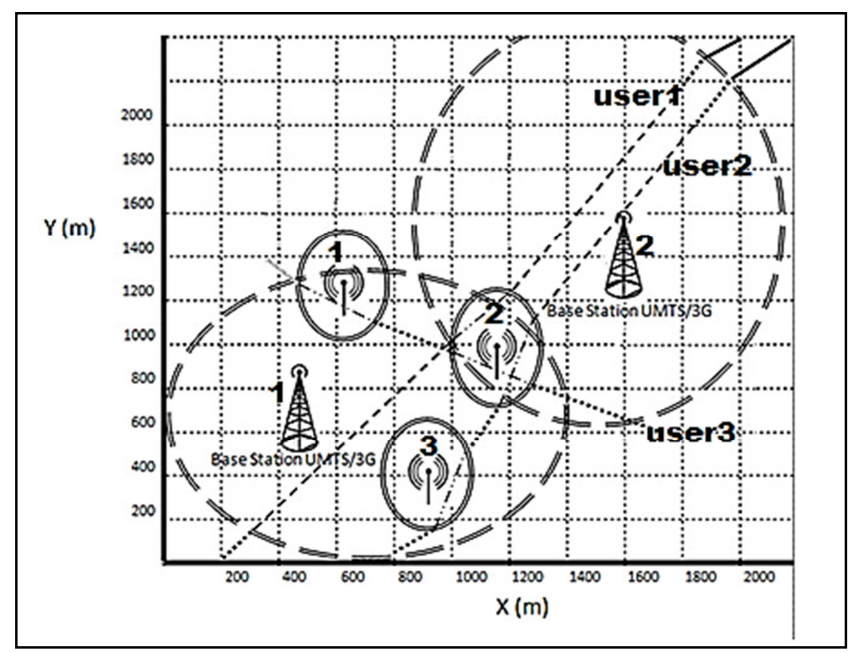

Figure 3 Simulation results $3 \mathrm{G}-\mathrm{WiFi}$ Offload with multiple base stations (proposed algorithm) $(*)$.

(*) The lines denote:

$$
\begin{aligned}
\cdots . .+ & \text { MS connection with } 3 \mathrm{G} \text { base stations in the range of }-75 \mathrm{dBm} \text { to }-95 \\
= & \mathrm{dBm} \\
-\cdots= & \text { MS connection with } \mathrm{WiFi} \mathrm{AP} \text { in the range of }-65 \mathrm{dBm} \text { to }-85 \mathrm{dBm} \\
--\cdot= & \text { MS connection with } \mathrm{WiFi} \mathrm{AP} \text { in the range of } 0 \mathrm{dBm} \text { to }-65 \mathrm{dBm} \\
& \mathrm{dBm}
\end{aligned}
$$

In Figure 2 we see that User3 is travelling from $(630,420)$ to $(500,480)$ and is not connected to the WiFi AP but served by the $3 \mathrm{G}$ BTS. This is because the WiFi traffic is in full state. In Figure 3 we notice that there is little difference incoverage for User3 compared to Figure 2. In Figure 3, User3 is connected to the WiFi AP, which indicates that the proposed algorithm is able to manage the traffic flow and stream traffic to another node when the node is in full or down state.

Furthermore, there is improvement regarding the handover time, as shown in Figure 4. 


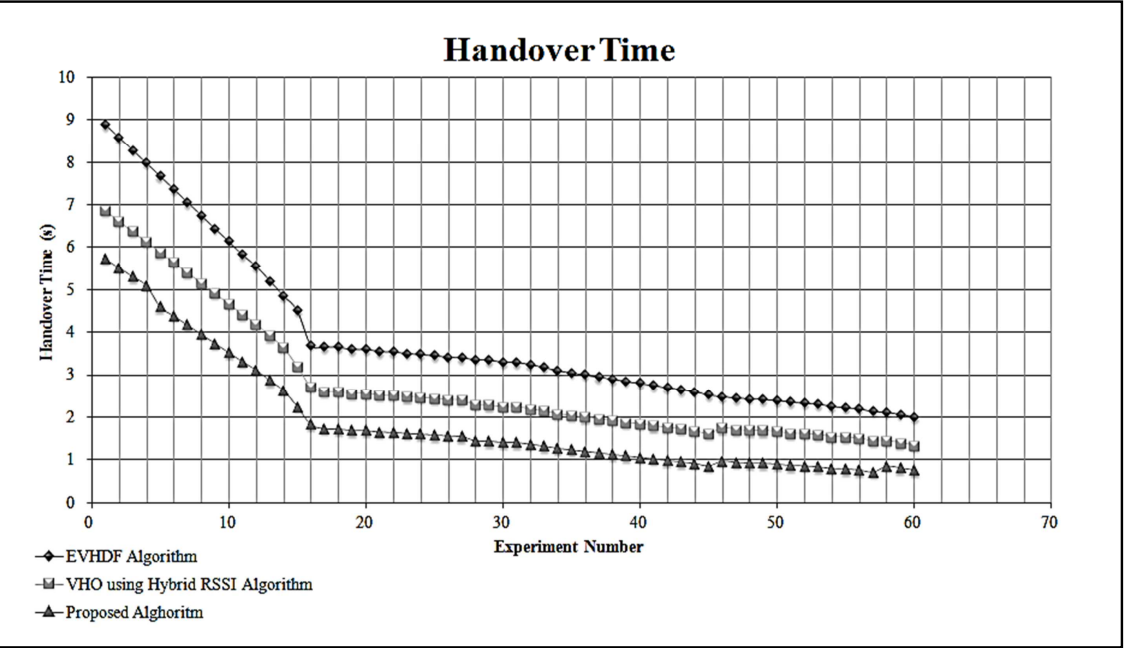

Figure 4 Handover Time.

From Figure 4, we can see that the proposed algorithm needs less time to execute handover because the GZRP makes the initialization time shorter with the track going through the smallest hop and the smallest traffic load.

In addition, when User3 is located in the coverage of WiFi AP2, there is a significant RSS level difference at the mark points 14-27, as shown in Figures 2 $\& 3$.

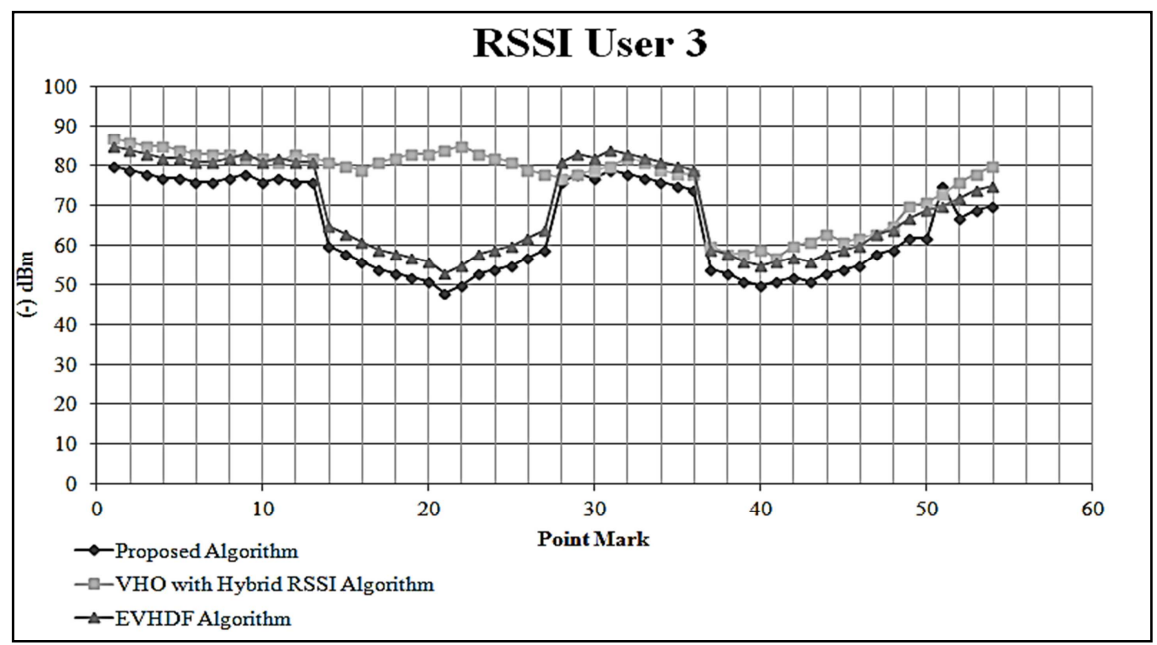

Figure 5 Graph of RSSI of User3. 
Figure 5 shows a comparison of RSSI between the proposed algorithm and other algorithms. From the figure we can see that a system using VHO with the Hybrid RSSI Algorithm yields an RSS level of around $-78 \mathrm{dBm}$ to $-85 \mathrm{dBm}$. This implicitly shows that the load for the AP is already full and the MS does not connect with the outside BTS. Unlike in the previous simulation, where we applied the proposed algorithm orthe EVHDF algorithm, the MS was successfully connected to the outside BTS. The RSS for the proposed algorithm is better than the RSS for the EVHDF, because network conditions can be well dispersed to various nodes. On the other hand, the EVHDF is not able to deploy traffic load balancing, because the EVHDF is only used to determine the condition of the network. With the proposed algorithm,User3 gets an RSS in the range of $-48 \mathrm{dBm}$ to $-60 \mathrm{dBm}$, while the RSS range with the EVHDF is -53 $\mathrm{dBm}$ to $-63 \mathrm{dBm}$. Thus, implementation of the GZRP in the $3 \mathrm{G}-\mathrm{WiFi}$ Offload affacted the quality of the RSS for the MS greatly.

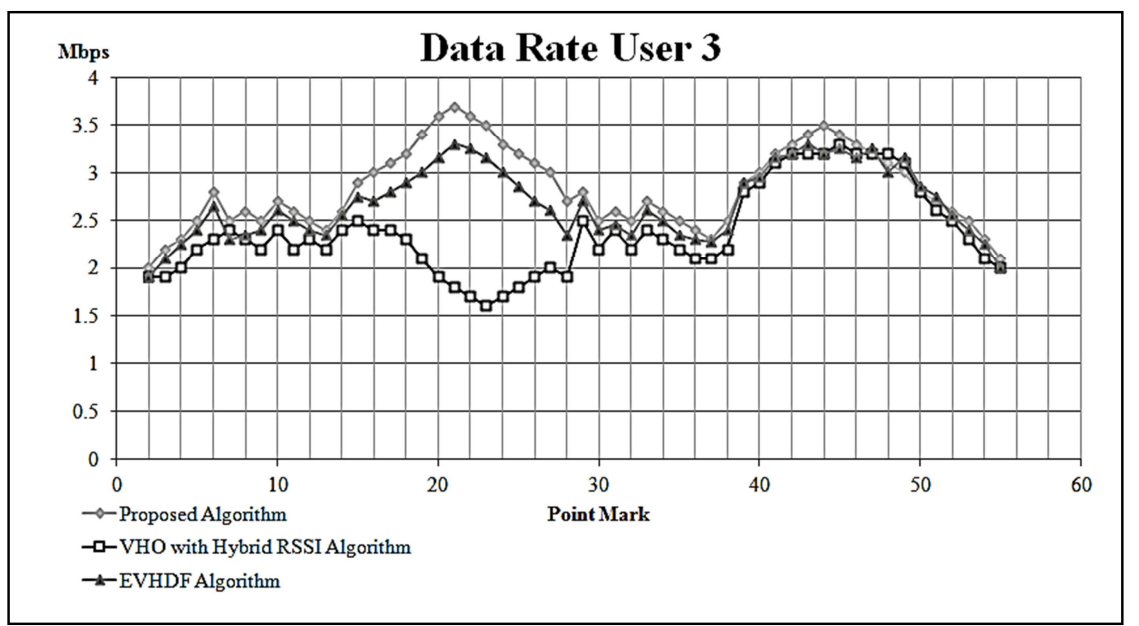

Figure 6 Graph of Data Rate of User3.

Figure 6 shows the results of the simulation regarding the data rate of User3 in WiFi AP2 coverage. We can see that there are some differences in the data rate between User3 using the proposed algorithm and the other algorithms. At the time when User1, User2, User3 are simultaneously in the coverage of WiFi AP2, they compete with each other to use the available bandwidth on the existing WiFi access. In the first simulation, User1 and User2 received the WiFi coverage first, after which it moved to User3, who received a WiFi coverage as good as User1 and User2, but User3 was still covered by the UMTS (3G) base station too, since handover to the WiFi network could not be performed. In the second simulation we used the proposed algorithm and the result was that for User3 handover could be performed from coverage by the UMTS (3G) base 
station to coverage by WiFi AP2. From Figure 6, the data rate of User3 had a similar gain as User1 and User2 because the existing traffic in the WiFi AP was supplied to a neighbor WiFi AP. The application of the GZRP yields an increase in data rate of $1 \mathrm{Mbps}$ to $2.5 \mathrm{Mbps}$

The total traffic of User3 in WiFi AP 2 is depicted in Figure 7, which shows that the total traffic for the proposed algorithm was lower than that of other the algorithms.

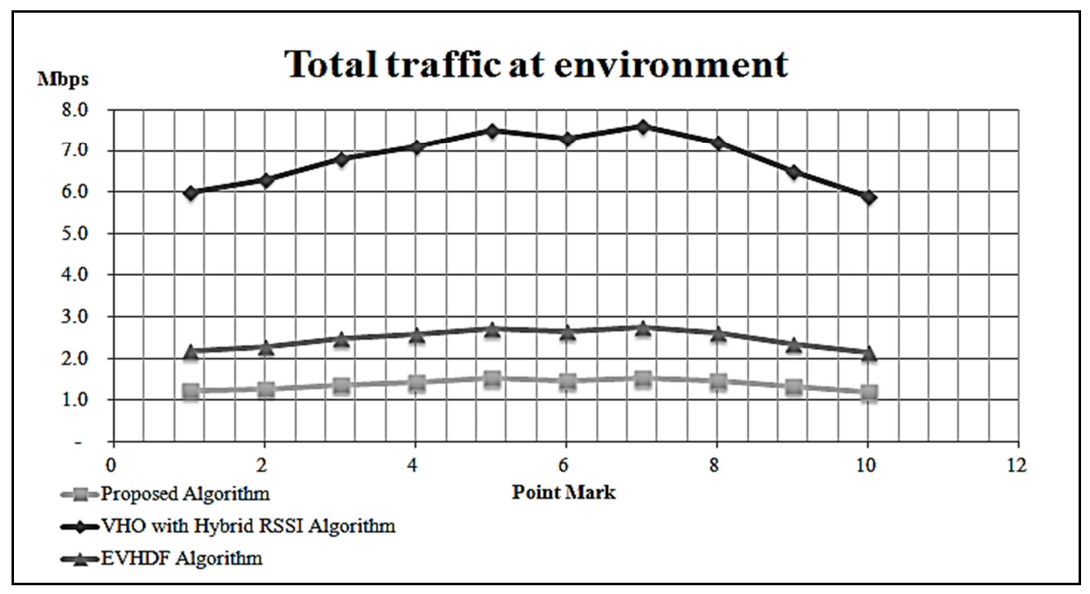

Figure 7 Total WiFi Traffic Capacity.

This proves that the traffic capacity at access point WiFi AP2 was streamed to the neighboring access points that were still connected to the same WiFi Offload gateway. The proposed algorithm was able to reduce the amount of traffic going to a bottleneck and minimized handover failures and call drop occurrences.

\section{Conclusions}

We have proposed a novel strategy for handover in 3G-WiFi Offload by combining the GZRP and the VHO algorithm. Based on the simulation results, we can conclude that there are significant improvements in terms of handover time, received signal strength, data rate, and traffic capacity. The results of our research have proved that a concept of dynamic routing in $3 \mathrm{G}-\mathrm{WiFi}$ offload technology with multiple base stations gives the best performance for the mobile subscriber since the GZRP is able to create a $3 \mathrm{G}-\mathrm{WiFi}$ offload network. As a result, providers and mobile network users will feel comfortable with the quality of the mobile network service. 


\section{Acknowledgements}

This work was partially supported by the Directorate General of Higher Education (DGHE) of the Republic of Indonesia. Part of the research was conducted at the South University of Science and Technology of China (SUSTC) under supervision of Prof. Yifan Chen. The authors also thank Filbert H. Juwono for his proofreading, comments, and suggestions.

\section{References}

[1] Cisco, Cisco Visual Networking Index: Global Mobile Data Traffic Forecast Update, 2010-2015, http://newsroom.cisco.com/dlls/ekits/ Cisco_VNI_Global_Mobile_Data_Traffic_Forecast_2010_2015.pdf, February 1, 2011. (15 March 2013)

[2] Chandrasekhar, V., Andrews, J.G. \& Gatherer, A., Femtocell Networks: A Survey, IEEE Communications Magazine, 46(9), pp. 59-67, 2008.

[3] Dimatteo, S., Hui, P., Han, B. \& Li, V.O.K., Cellular Traffic Offloading through WiFi Networks, $8^{\text {th }}$ IEEE International Conference on Mobile Adhoc and Sensor Systems (MASS), pp. 192-201, 17-22 Oct. 2011.

[4] Busanelli, S., Martalo, M., Ferrari, G. \& Spigoni, G., Vertical Handover between WiFi and UMTS Networks: Experimental Performance Analysis, International Journal of Energy, Information and Communications, 2(1), pp. 75-96, 2011.

[5] Marquez-Barja, J., Calafate, C.M.T., Cano, J.C. \& Manzoni, P., An Overview of Vertical Handover Techniques: Algorithms, Protocols and Tools. Computer Communication, 34, pp. 985-987, 2011.

[6] Haas, J., A New Routing Protocol for the Reconfigurable Wireless Networks, Proc. IEEE Int. Conf. on Universal Personal Communications, pp. 562-566, 1997.

[7] Haas, Z.J., Pearlman, M.R. \& Samar, P., Intrazone Routing Protocol (IARP), IEFT internet Draft, http://tools.ietf.org/html/draft-ietf-manetzone-iarp-01, June 2001. (19 January 2013)

[8] Haas, Z.J., Pearlman, M.R. \& Samar, P., Bordercast Resolution Protocol (BRP), IEFT Internet Draft, http://tools.ietf.org/html/draft-ietf-manetzone-brp-01, June 2001. (23 January 2013)

[9] Goldberg, D.E., Genetic Algorithms in Search, Optimization \& Machine Learning, Reading, MA: Addison-Wesley, 1989.

[10] Kumar, R.S. \& Ramachandram, S., Genetic Zone Routing Protocol, Int. J. of Theoretical and Appl. Info. Tech., 4(9), pp. 789-794, 2008.

[11] Kumar, R.S. \& Ramachandram, S., Scalability of Network Size on Genetic Zone Routing Protocol for MANETs, International Conference on Advanced Computer Theory and Engineering, pp. 763-767, 2008. 
[12] Nikhil, K., Agarwal, S. \& Sharma, P., Application of Genetic Algorithm in Designing a Security Model for Mobile Adhoc Network, Department of IT, ABES Engineering College, Ghaziabad (UP), India, 2012

[13] Kumar, R.S. \& Ramachandram, S., The Performance Evaluation of Cached Genetic Zone Routing Protocol for MANETs, 16th IEEE International Conference on Networks, 2008.

[14] Nasser, N., Hasswa, A. \& Hassanein, H., Handoffs in Fourth Generation Heterogeneous Network, IEEE Communication Magazine, 44(10), pp. 96-103, 2006.

[15] He, D., Chi, C., Chan, S., Chen, C., Bu, J. \& Yin, M., A Simple and Robust Vertical Handoff Algorithm for Heterogeneous Wireless Mobile Networks, Wireless Personal Communications, 59(2), pp. 361-373, 2010.

[16] Kumar, R.S. \& Ramachandram, S., Load Balancing in Genetic Zone Routing Protocol for MANETs, International Journal of Computer and Information Engineering, 3(4), pp. 261-266, 2009.

[17] Anjum, A., Mohammed \& Nagib, G., Optimal Routing In Ad-Hoc Network Using Genetic Algorithm, Int. J. Advanced Networking and Applications, 3(5), pp. 1323-1328, 2012.

[18] Budiyanto, S., Asvial, M. \& Gunawan, D., Implementation of Genetic Zone Routing Protocol (GZRP) in 3G-WiFi Offload Multi Base Station, Intl. Conference of IEEE Tencon 2013, Xi' an-China, Oct 2013.

[19] Zander, J., Kim, S-L., Almgren, M. \& Queseth, O., Radio Resource Management for Wireless Networks, Artech House, Inc., Norwood, MA, USA, 2001.

[20] Yoo, S., Cypher, D. \& Golmie, N., Timely Effective Handover Mechanism in Heterogeneous Wireless Networks, Wireless Personal Communications, 52(3), pp. 449-475, 2010. 